\title{
MODELLING OF TIME-DEPENDENT BEHAVIOUR OF PARTICULATE THERMOSET POLYMERS
}

\author{
JAN VOZÁB*, JAN VOREL \\ Czech Technical University in Prague, Faculty of Civil Engineering, Department of Mechanics, Thákurova 7, \\ 16629 Prague 6, Czech Republic \\ * corresponding author: jan.vozab@fsv.cvut.cz
}

\begin{abstract}
A preliminary study of a numerical model describing the behaviour of polymer-based composites is presented. The numerical model consists of three main parts. The first is the microplane M4 model, which is the main part of the model and is used to simulate elastoplastic behaviour and damage. The second part consists of a generalized Maxwell model, which adds the effect of linear creep of the material to the calculation. The last part is a free volume model that extends the linear creep to the nonlinear creep. The creep is calculated on the deviatoric part of the normal stress of each microplane, which allows the model to capture the polymer behaviour adequately without adjusting the free volume of the model.
\end{abstract}

KEYWORDS: Creep, free volume, microplane, thermosets, viscoelasticity.

\section{INTRODUCTION}

Many different materials are used in building constructions. One specific type that is increasingly used is epoxies. These polymers are often used to form different types of composites, as universal adhesives, rigid foams, or as structural adhesives [1]. Like many polymeric materials, they are subject to nonlinear creep, which is associated with susceptibility to changes in temperature and humidity [2-4].

To properly design components made of this material, the appropriate numerical tool is essential. Many models, describing broad nonlinear time-dependent responses of these material types, have been developed $[5,6]$. By combining these approaches with the generally accepted material model for mechanical loading, the overall material response can be adequately described.

In this paper, we introduce the novel approach combining a microplane material model M4 [7, 8], which is enhanced with the free volume approach on the microplane level to capture the time-dependent behaviour [5].

\section{NUMERICAL MODEL}

Simulating nonlinear behaviour is challenging because no nonlinear theory has been introduced that can describe all materials. However, many models have been developed that describe broad nonlinear responses $[5,6]$. By combining these approaches, it is then possible to describe the behaviour of these materials. The numerical model described in this paper consists of three main parts. The microplane material model M4 [7, 8] with improvements presented in [9] is used as a fundamental part. The Maxwell chain on the microplane level is used to capture the creep behaviour [10]. The additional free volume approach [5] allows for describing nonlinear viscoelastic behaviour under small to moderate strains.

\subsection{Microplane Model M4}

The microplane material model M4 [7] is the fourth version of the microplane model introduced by Bažant $[11,12]$. This constitutive model is characterized by the relationships between stresses and strains projected onto a surface of a microplane, which is oriented by its own normal vector. The basic idea is that the strain vector $\overrightarrow{\varepsilon_{N}}$ on the microplane is a projection of a strain tensor $\varepsilon$. Then the normal strain on the microplane have the form

$$
\varepsilon_{N}=N_{i j} \varepsilon_{i j}
$$

Similarly, the shear components, which are characterized in the direction $M$ and $L$, are given by orthogonal vectors, $\vec{m}$ and $\vec{l}$, take the form

$$
\varepsilon_{M}=M_{i j} \varepsilon_{i j} ; \quad \varepsilon_{L}=L_{i j} \varepsilon_{i j} .
$$

in which $M_{i j}=\left(m_{i} n_{j}\right)+\left(m_{j} n_{i}\right) / 2$ and $L_{i j}=\left(l_{i} n_{j}+\right.$ $\left.l_{j} n_{i}\right) / 2[13,14]$. All three components can be seen in Fig.1(a).

The static equivalence is computed approximately by the principle of virtual work written for the surface $\Omega$ of a unit hemisphere

$$
\frac{2 \pi}{3} \sigma_{i j} \delta \varepsilon_{i j}=\int_{\Omega}\left(\sigma_{N} \delta \varepsilon_{N}+\sigma_{L} \delta \varepsilon_{L}+\sigma_{M} \delta \varepsilon_{M}\right) d \Omega .
$$

This equation represents that the virtual work of macro-stresses within a unit sphere must be equal to the virtual work of micro-stresses regarded as the tractions on the surface of the sphere [7]. Substituting $\delta \varepsilon_{N}=N_{i j} \delta \varepsilon_{i j}, \delta \varepsilon_{M}=M_{i j} \delta \varepsilon_{i j}$ and $\delta \varepsilon_{L}=L_{i j} \delta \varepsilon_{i j}$, and noting that the last variational equation must 


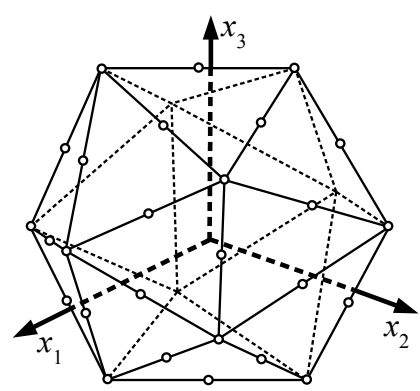

(a)

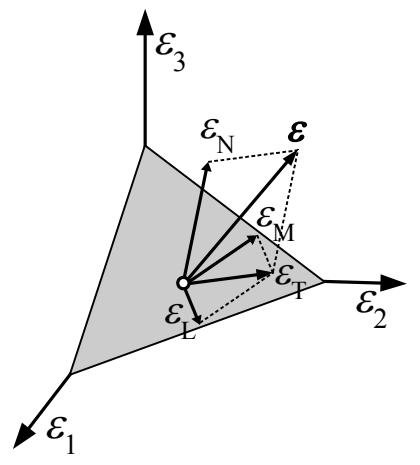

(b)

Figure 1. Microplane model: (a) distribution of integration points (microplane normals) - system of 21 microplanes per hemisphere; (b) microplane strain components

include any variation $\delta \varepsilon_{i j}$, the result is the following basic equilibrium equation [12]:

$$
\sigma_{i j}=\frac{3}{2 \pi} \int_{\Omega} s_{i j} d \Omega \approx 6 \sum_{\mu=1}^{N_{m}} w_{\mu} s_{i j}^{(\mu)},
$$

with

$$
s_{i j}=\sigma_{N} N_{i j}+\sigma_{M} M_{i j}+\sigma_{L} L_{i j} .
$$

This integral is an approximation by an optimal Gaussian integration formula for a spherical surface, as you can see in Fig.1(b). This surface is representing a weighted sum over the microplanes. In the finite element algorithms, this integral has to be computed at each integration point of each finite element in each time step [7].

The most generalized constitutive relations on the microplane level have the forms

$$
\begin{aligned}
\sigma_{N}(t) & =\mathcal{F}_{\tau=0}^{t}\left[\varepsilon_{N}(\tau), \varepsilon_{M}(\tau), \varepsilon_{L}(\tau)\right], \\
\sigma_{L}(t) & =\mathcal{G}_{\tau=0}^{t}\left[\varepsilon_{N}(\tau), \varepsilon_{M}(\tau), \varepsilon_{L}(\tau)\right], \\
\sigma_{M}(t) & =\mathcal{H}_{\tau=0}^{t}\left[\varepsilon_{N}(\tau), \varepsilon_{M}(\tau), \varepsilon_{L}(\tau)\right],
\end{aligned}
$$

where $\mathcal{F}, \mathcal{L}$ and $\mathcal{H}$ are functionals of the microplane strains in time $t$.

Volumetric/deviatoric decomposition of the normal strain component [15] needs to be employed to capture the Poisson ratio of polymers adequately

$$
\varepsilon_{D}=\varepsilon_{N}-\varepsilon_{v} ; \varepsilon_{v}=\varepsilon_{k k} / 3,
$$

where $\varepsilon_{v}$ stands for the volumetric strain and $D$ denotes the deviatoric component.

\subsection{MAXWELL CHAIN}

The formulation of the microplane model M4 briefly described in the previous section is extended to incorporate time-dependent behaviour of epoxies. Approach similar to [10] is utilized. Creep is developed at every integration point, i.e. on each microplane of every integration point. On the level of microplanes, creep is implemented as linearly viscoelastic.

The behavior of each Maxwell element $(\mu)$, where a spring and a dashpot are connected in series, is expressed as

$$
\dot{\varepsilon_{\mu}}=\frac{\dot{\sigma_{\mu}}}{E_{\mu}}+\frac{\sigma_{\mu}}{\eta_{\mu}}
$$

where $E_{\mu}$ is the elastic modulus and $\eta_{\mu}$ is the viscosity of the element. In the proposed model, the viscosity is assumed only for the deviatoric part of the normal strain component on each microplane separately. Thus, according to the approximation by the generalized Maxwell model, the equation for calculation of deviatoric stress predictor in the incremental form reads

$$
\begin{aligned}
\sigma_{D}^{v e} & =\sigma_{D}^{i}+E_{D}^{\prime \prime}\left(\varepsilon_{D}-\varepsilon_{D}^{i}\right)-\Delta \sigma_{D}^{\prime \prime}= \\
& =\sigma_{D}^{i}+E_{D}^{\prime \prime}\left(\Delta \varepsilon_{D}-\Delta \varepsilon_{D}^{\prime \prime}\right)
\end{aligned}
$$

where $i$ means the initial value in the time step $\Delta t$ and ve stands for the viscoelastic stress increment, which is the generalization of the elastic stress increment. Variables labeled by " are calculated according to the equations

$$
\begin{aligned}
E_{D}^{\prime \prime} & =\sum_{\mu=1}^{N} \frac{1-e^{-\Delta t / \tau_{\mu}}}{\Delta t / \tau_{\mu}} E_{D, \mu}, \\
\Delta \sigma_{D}^{\prime \prime} & =\sum_{\mu=1}^{N}\left(1-e^{-\Delta t / \tau_{\mu}}\right) \sigma_{D, \mu}^{i}, \\
\Delta \varepsilon_{D}^{\prime \prime} & =\Delta \sigma_{D}^{\prime \prime} / E_{D}^{\prime \prime},
\end{aligned}
$$

where $N$ is the number of Maxwell elements and $\tau_{\mu}=$ $\eta_{\mu} / E_{D, \mu}$ relaxation time of the $\mu$-th element. If a constant Poisson's ratio $\nu$ is assumed

$$
E_{D, \mu}=\frac{5 E_{\mu}}{(2+3 \gamma)(1+\nu)}
$$

where $\gamma$ is a parameter that may be chosen or can be optimized so as to match the given test data best (this value is set to 1 in this study) [16]. Note that remaining constitutive relations remain unchanged and the moduli are updated based on $E^{\prime \prime}=E_{D}^{\prime \prime}(2+3 \gamma)(1+\nu)$.

\subsection{Free volume}

The last extension of our model is a free volume approach to extend the viscoelastic behaviour to a nonlinear scale, which is more convenient for the material. The description of this approach can be found 


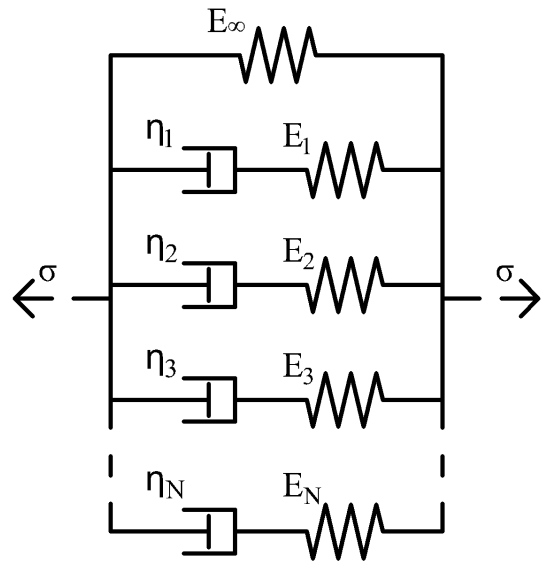

Figure 2. Reological model of the Maxwell chain.

\begin{tabular}{ccc}
\hline & $\tau_{\mu}$ & $E_{\mu}[\mathrm{MPa}]$ \\
\hline 1 & 10 & 2.6174 \\
2 & $10^{2}$ & 107.5458 \\
3 & $10^{3}$ & 455.0239 \\
4 & $10^{4}$ & 933.0051 \\
5 & $10^{5}$ & 562.4520 \\
6 & $10^{6}$ & 273.8973 \\
7 & $10^{7}$ & 198.4917 \\
8 & $10^{8}$ & 135.9774 \\
9 & $10^{9}$ & 41.7351 \\
10 & $10^{10}$ & 4.0175 \\
11 & $10^{11}$ & 4.7209 \\
12 & $10^{12}$ & -0.0819 \\
13 & $\infty$ & 39.9691 \\
\hline
\end{tabular}

TABLE 1. Used values of Maxwell chain. Values converted from [5, Tables 1 and 2].

in [5]. In the employed approach, the dilation is introduced into the time scale, which results in a nonlinear system of equations. Note that for the case of infinitesimal dilatation, the nonlinear free volume theory reverts to linear viscoelasticity. In general, temperature $(T)$, moisture content $(c)$ and mechanical dilatation $(\theta)$ influence the time scale of the material in a similar manner such that

$$
a=a(T, c, \theta) .
$$

The shift factor $a$ is connected to the free volume as suggested in $[17,18]$

$$
\log _{10} a=\frac{B}{2.303}\left(\frac{1}{f}-\frac{1}{f_{0}}\right),
$$

where $f$ is fractional free volume, $f_{0}$ represent the fractional free volume at a reference state. The coupling of the free volume to material parameters that affect its macroscopic volume can be expressed using a linear dependence

$$
f=f_{0}+\alpha_{v} \Delta T+\beta_{v} \Delta c+\delta \theta,
$$

where $\alpha_{v}$ is the volumetric thermal expansion of the free volume, $\beta_{v}$ represent the volumetric expansion due to a change in moisture content and $\delta$ relates the change if the free volume due to mechanical volume changes of the polymer. Combining Eq. (17) with Eq. (18) we get

$$
\log a=\frac{-B}{2.303 f_{0}}\left(\frac{\alpha_{v} \Delta T+\beta_{v} \Delta c+\delta \theta}{f_{0}+\alpha_{v} \Delta T+\beta_{v} \Delta c+\delta \theta}\right) .
$$

This equation includes the influence of all essential properties related to the development of the volume changes. The influence of the shift factor on the stress response is through convolution, or superposition, integrals relating the time dependent stress response to the strain history [5] by

$$
\sigma_{D}=\int_{0}^{t} E_{D}^{\prime \prime}\left(t^{\prime}-\tau^{\prime}\right) \frac{\partial \varepsilon_{D}}{\partial \tau} d \tau
$$

where the kernel takes the form

$$
t^{\prime}-\tau^{\prime}=\int_{\tau}^{t} \frac{d \xi}{a[T(\xi), c(\xi), \theta \xi]}
$$

to account for the temperature, moisture and dilatation histories. Note that in [5], the modified free volume approach is utilized to simulate shear-dominated loading scenarios with little or no dilatation. Such an approach is not needed in the current formulation since the creep behaviour is defined on microplanes.

\begin{tabular}{cc}
\hline Parameter & Value \\
\hline$B$ & 0.5 \\
$f_{0}$ & 0.1 \\
$\delta$ & 1 \\
$\alpha_{v}$ & $2.857 \times 10^{-3}{ }^{\circ} \mathrm{C}^{-1}$ \\
$T_{0}$ & $55{ }^{\circ} \mathrm{C}$ \\
\hline
\end{tabular}

TABLE 2. Used material parameters. Values taken from $[5]$.

\section{Results}

In this phase of the development process, we used a single-point calculation to verify the numerical model using [19]. The values for the Maxwell chain from Table 1 and the material parameters from Table 2 were used in the simulation. The element was loaded by pure shear deformation. Fig. 3 shows the force vs. displacement diagram. The blue line represents the elastic loading of the element, and it represents the upper bound for the presented results. The red line shows the elastic loading of the material, including the effect of the Maxwell chain. As expected, creep affects the magnitude of the reaction, and therefore the curve has a smaller slope. The last green line represents the investigated microplane model. Initially, it has the same inclination as the elastic model with the Maxwell chain, but as the deformation increases, the response deviates. Note that for this study, the microplane model behaviour is assumed to be purely elastic. 


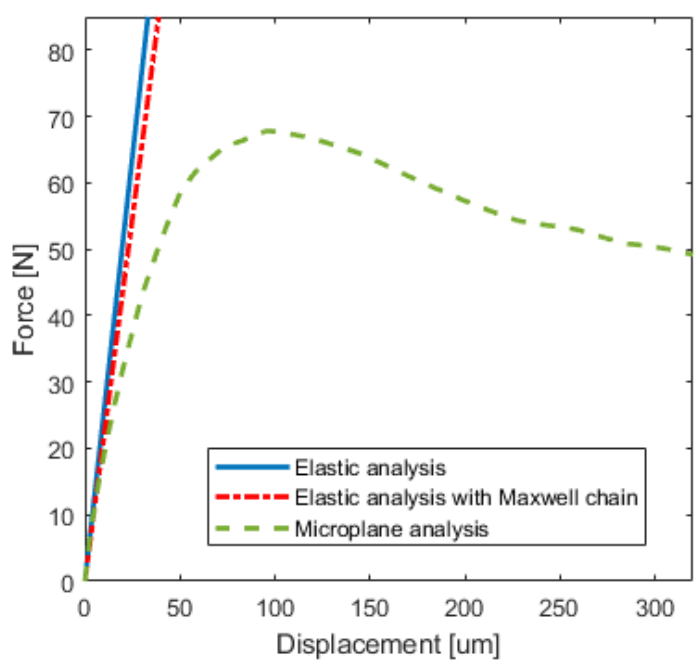

Figure 3. Results from finite element analysis: Cube with an edge $1 \mathrm{~cm}$, loading pure shear.

\section{Conclusions}

This paper presented a new model that can be used to describe the nonlinear creep of thermosets, which is composed of three parts: (i) the microplane model M4, which calculates elastoplasticity and damage; (ii) the Maxwell chain model, which adds the effect of linear creep to the calculation; (iii) the free volume model, which extends creep to a nonlinear scale. In Fig.3, we can see that according to preliminary results, the model behaves as expected because the effect of creep and softening is noticeable under shear load. At the same time, it is necessary to test the model on a larger scale at different load cases to ensure its accuracy. The Arcan experiment presented in [5] would be appropriate. Furthermore, it is necessary to test the model under a combination of different temperatures and humidity for verification of the free volume model parameters. If the accuracy of the model is still not sufficient, it could be caused by curing of the material which could be additionally connected to our numerical model.

\section{ACKNOWLEDGEMENTS}

The financial support provided by the GAČR grant No. 19-15666S and by the Czech Technical University in Prague within SGS project with the application registered under the No. SGS20/155/OHK1/3T/11 is gratefully acknowledged.

\section{REFERENCES}

[1] M. A. Meyers, K. K. Chawla. Mechanical Behavior of Materials. Cambridge University Press, 2nd edn., 2008.

[2] J.-P. Pascault, H. Sautereau, J. Verdu, R. J. Williams. Thermosetting polymers, vol. 64. CRC Press, 2002.

[3] R. F. Landel, L. E. Nielsen. Mechanical properties of polymers and composites. CRC press, 1993.

[4] P. Silva, T. Valente, M. Azenha, et al. Viscoelastic response of an epoxy adhesive for construction since its early ages: Experiments and modelling. Composites Part B: Engineering 116:266-277, 2017.

[5] C. F. Popelar, K. M. Liechti. Multiaxial Nonlinear Viscoelastic Characterization and Modeling of a Structural Adhesive. Journal of Engineering Materials and Technology 119(3):205-210, 1997.

https://asmedigitalcollection.asme.org/ materialstechnology/article-pdf/119/3/205/ 5681124/205_1.pdf DOI:10.1115/1.2812245.

[6] G. Cusatis, D. Pelessone, A. Mencarelli. Lattice discrete particle model (ldpm) for failure behavior of concrete. i: Theory. Cement and Concrete Composites 33(9):881-890, 2011.

[7] Z. P. Bažant, F. C. Caner, I. Carol, et al. Microplane model $\mathrm{m} 4$ for concrete. i: Formulation with work-conjugate deviatoric stress. Journal of Engineering Mechanics 126(9):944-953, 2000.

[8] F. C. Caner, Z. P. Bažant. Microplane model $\mathrm{m} 4$ for concrete. ii: Algorithm and calibration. Journal of Engineering Mechanics 126(9):954-961, 2000.

[9] G. Di Luzio. A symmetric over-nonlocal microplane model $\mathrm{m} 4$ for fracture in concrete. International journal of solids and structures 44(13):4418-4441, 2007.

[10] Z. P. Bažant, F. C. Caner, M. D. Adley, S. A. Akers. Fracturing rate effect and creep in microplane model for dynamics. Journal of engineering mechanics 126(9):962-970, 2000.

[11] Z. Bažant. Microplane model for strain-controlled inelastic behaviour, chapter 3. Mechanics of engineering materials, chapter 31984.

[12] Z. P. Bažant, P. G. Gambarova. Crack shear in concrete: Crack band microplane model. Journal of Structural Engineering 110(9):2015-2035, 1984.

[13] Z. P. Bažant, P. C. Prat. Microplane model for brittle-plastic material: I. theory. Journal of Engineering Mechanics 114(10):1672-1688, 1988.

[14] Z. P. Bažant, P. C. Prat. Microplane model for brittle-plastic material: Ii. verification. Journal of Engineering Mechanics 114(10):1689-1702, 1988.

[15] I. Carol, Z. Bažant. Damage and plasticity in microplane theory. International Journal of Solids and Structures 34(29):3807-3835, 1997.

[16] Z. Bažant, Y. Xiang, P. Prat. Microplane model for concrete. I: Stress-strain boundaries and finite strain. Journal of Engineering Mechanics 122(3):245-254, 1996.

[17] A. K. Doolittle. Studies in newtonian flow. ii. the dependence of the viscosity of liquids on freeâĂ $\check{\text { Rspace. }}$ Journal of Applied Physics 22(12):1471-1475, 1951. https://doi.org/10.1063/1.1699894 DOI:10.1063/1.1699894.

[18] A. K. Doolittle, D. B. Doolittle. Studies in newtonian flow. v. further verification of the freeâǍrspace viscosity equation. Journal of Applied Physics 28(8):901-905, 1957. https://doi.org/10.1063/1.1722884 DOI:10.1063/1.1722884.

[19] ES3. Mars - modeling and analysis of the response structures. 